\title{
HIGH-PRECISION PERFORMANCE TESTING OF THE LHC POWER CONVERTERS
}

\author{
M. Bastos, A. Cantone, P. Dreesen, G. Fernqvist, O. Fournier, G. Hudson \\ CERN, Geneva, Switzerland
}

\begin{abstract}
The magnet power converters for LHC were procured in three parts, power part, current transducers and control electronics, to enable a maximum of industrial participation in the manufacturing and still guarantee the very high precision (a few parts in $10^{-6}$ ) required by LHC. One consequence of this approach was several stages of system tests: factory reception tests, CERN reception tests, integration tests , short-circuit tests and commissioning on the final load in the LHC tunnel. The majority of the power converters for LHC have now been delivered, integrated into complete converter and high-precision performance testing is well advanced. This paper presents the techniques used for high-precision testing and the results obtained.
\end{abstract}

CERN, 


\title{
HIGH-PRECISION PERFORMANCE TESTING OF THE LHC POWER CONVERTERS
}

\author{
M. Bastos, A. Cantone, P. Dreesen, G. Fernqvist, O. Fournier, G. Hudson, CERN, Geneva, \\ Switzerland
}

\begin{abstract}
The magnet power converters for LHC were procured in three parts, power part, current transducers and control electronics, to enable a maximum of industrial participation in the manufacturing and still guarantee the very high precision (a few parts in $10^{-6}$ ) required by LHC. One consequence of this approach was several stages of system tests: factory reception tests, CERN reception tests, integration tests, short-circuit tests and commissioning on the final load in the LHC tunnel. The majority of the power converters for LHC have now been delivered, integrated into complete converters and highprecision performance testing is well advanced. This paper presents the techniques used for high-precision testing and the results obtained.
\end{abstract}

\section{INTRODUCTION}

The LHC collider requires an unprecedented precision performance from its magnet power converters. The power converter design, the control strategy and its rationale have been described many times before. The design of the converters and their control included a strategy to maximise an industrial participation whilst obtaining the very high accuracy, ppm $\left(10^{-6}\right)$ level, dictated by the LHC requirements [1]. As a result the design and manufacturing was adapted for each subsystem to the availability of industrial partners and many companies have been delivering subsystems to CERN, where they were combined into the final power converters. An extensive system of progressive testing and quality control had to be in place to guarantee the final performance. Below are listed some of the key precision parameters specified and tested.

\section{Static performance:}

DC accuracy at a given temperature

Noise, 0.1 - $10 \mathrm{~Hz}$ bandwidth (BW)

Stability with temperature and time, $0.5-100 \mathrm{mHz}$ BW

Reproducibility

Susceptibility to interference (EMC)

Dynamic performance:

Control loop settling behaviour/stability

Thermally induced settling behaviour

Tracking error with respect to the reference input value

The high-precision current control performance in the LHC configuration is mainly determined by the DC current transducer (DCCT), the analogue-digital converter (ADC) and the control loop. To optimise design and cost, five different classes of DCCTs and four different types of ADCs are used [1]. All the DCCTs were made by industry, but with substantial support from CERN. The ADCs and the control loops were designed at CERN and manufactured by industry.

\section{TEST STRATEGY}

The LHC collider is a tremendous technical challenge in many technological domains, not the least in power conversion. To help reduce the difficulties in commissioning and operating LHC, all power converters and their subsystems were subjected to very thorough performance tests several times, also to ensure a very high reliability.

Equipment was first tested in the factory, but also on arrival at CERN. The subsystems were then all integrated in complete power converters and the overall performance was tested. The complete converter was stored until installation time in the underground tunnel (could be many months later). After installation, the converter was connected in its final configuration, but the magnet load was replaced with a short-circuit. A full functional test was carried out as well as a re-calibration of the DCCT and the ADC. The final step was to connect the final superconducting load and verify several key parameters again.

Most precision tests were performed with automatic testbeds and computer control to ensure that all tests were carried out and in a reproducible and efficient fashion.

\section{FACTORY RECEPTION TESTS}

All equipment was specified to be fully tested by the manufacturers and in general they proposed test programmes to CERN for approval. In many instances some parameters could not be tested in the factory due to lack of test facilities or equipment. A significant portion, 5-25\% depending on type, of the equipment arriving at CERN did not comply fully with the demanding highperformance specification.

\section{RECEPTION TESTS AT CERN}

\section{DCCTs}

All DCCTs, were tested in special testbeds on arrival at CERN. The 6kA and 20kA testbeds could take four DCCTs in parallel and the 600A, fig. 1, could take eight. Key parameters were chosen as a result of pre-series testing. The rejection rate for DCCTs was $>5 \%$ (mainly precision parameters) and decreased little between production batches.

T11 Power Supplies 


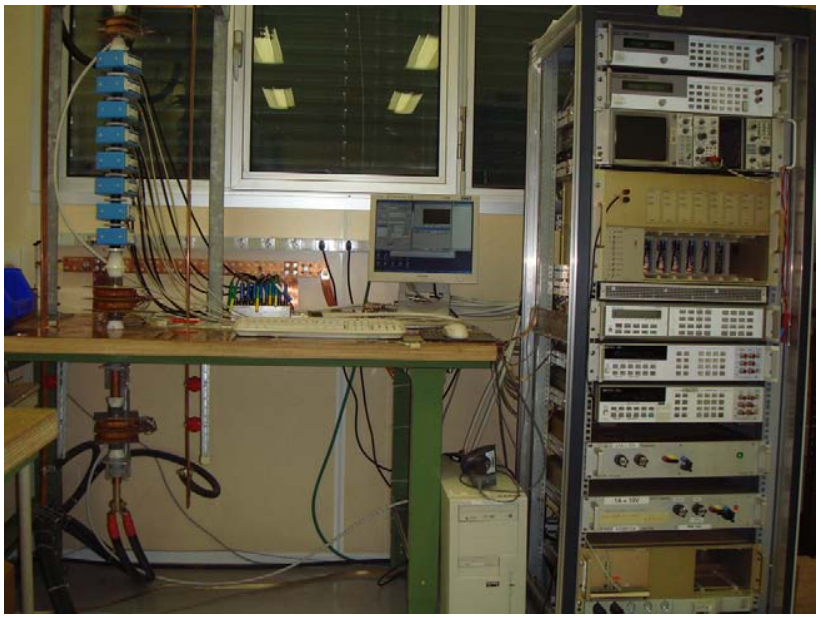

Fig. 1: Testbed for DCCTs up to $600 \mathrm{~A}$

The following table gives an overview of the test conditions and results:

Table 1: Overview of DCCT tests

\begin{tabular}{|l|l|l|l|}
\hline Type & $4-13 \mathrm{kA}$ & $600 \mathrm{~A}$ & $120 \mathrm{~A}$ \\
\hline Quantity & 450 & 1050 & 2450 \\
\hline Time/DCCT & 8 hours & 4 hours & 2 hours \\
\hline Nr of param & 30 & 30 & 23 \\
\hline Reject rate & $4.7 \%$ & $7.2 \%$ & $12.5 \%$ \\
\hline Precision class & $2 \mathrm{ppm}$ & $10 \mathrm{ppm}$ & $100 \mathrm{ppm}$ \\
\hline
\end{tabular}

Most problems with 600A and 120A were concerned with settling times and temperature related drifts and could be traced back to burden resistor performance limitations. It is in fact difficult and time-consuming to test the burden and manufacturers do normally not do it.

The CERN testbeds proved their worth in testing such a large quantity of DCCTs to an unprecedented precision.

\section{FGCs/ADCs}

The FGCs (2000 units) were given a functional test and then a 3 week burn-in. In the first delivery batch about $25 \%$ failed the functional test, but quality improved and later batches had 3-4\% failure rate.

\section{INTEGRATION TESTS AT CERN}

The precision tests are the last of a sequence that includes functional tests, EMC tests and thermal tests. For each sequence, the converter is placed in a custom designed test bed and connected to a test load, typically $1 \mathrm{~m} \Omega, 1 \mathrm{mH}$, via an external reference DCCT.

As mentioned before, the accuracy and reproducibility of the converters are mainly determined by the DCCT and the ADC performance. To guarantee optimal performance, special instrumentation is needed to calibrate and evaluate these precision components:

- CERN DCCT Calibrator (CDC) and Voltage Booster (VB) is a 24-bit programmable current reference [2].
- A 10V reference (Metron PBC) and a DVM (Agilent 3458) is used to calibrate the 22-bit and 16-bit ADCs

- A group of selected and specially improved highcurrent DCCTs are used as reference DCCTs. They are located in separate racks for best performance.

- Temperature probes

- A standards laboratory guaranteeing the calibration of thes instruments to better than $1 \mathrm{ppm}$.

Before each test, the converter DCCTs and ADCs are calibrated. An automatic test program then uses a reference DCCT and DVM to evaluate the converter performance by running tests on stability, noise, settling time, linearity, gain and offset errors, repeatability and reproducibility.

The integration tests with production power converters started early 2005. The first results were used to refine the performance limits set in the test program and identify systematic problems. The difficulties found during this phase [3] led to parallel investigations aimed at finding the best solution for each problem. The main problems and their remedies are listed below.

- Temperature dependent drift - The initial tests on the power converters with internal ADCs showed a $1 / 2$ hour-stability exceeding the limit of $3 \mathrm{ppm}$. It was soon clear that it was mainly temperature dependent and that both the DCCT and ADC were involved. An air deflector was introduced inside the DCCT electronics and a fan tray added to each FGC chassis for better ventilation of the precision components (ADCs, voltage references, DCCT burden resistor). In parallel, given the importance of the ADC drift, another test campaign characterized the temperature behaviour of the ADC system. This investigation led to the implementation of a temperature drift compensation algorithm for both the ADCs and the DCCTs [4].

- Noise - The initial tests showed a higher than expected noise level, 2-10 times depending on type. Many reasons were possible, but difficulties in loop tuning and high sensitivity to loop parameters pointed in the direction of control loop behaviour. This led to modifications in the implementation of the regulation algorithm [4]. The immunity of the converter electronics to external interferences was not sufficient and the system needed improved EMC shielding.

- Testbed instrumentation was too sensitive to EMI, jumps of 10-100ppm, mainly caused by the powerful radio station used by the CERN Fire Brigade. More shielding and the use of ferrite toroids along supply and signal cables resolved this problem.

- 600A and 120A testbeds proved sensitive to vibrations. Jumps of tens of ppms were caused by varying resistance in the busbar connections and the contactors used in the load circuit to configure the load. Recommendations not to move heavy objects 
around the converter during high-precision tests removed the problem.

The production tests of the converters continued in parallel with the investigations described in the previous section. This meant that neither the temperature correction algorithm, nor the loop modifications were ready at the start of the series testing of the converters. The results of the integration tests, table 1 , are expressed in ppm, mean value $\pm 1 \sigma$

Table 1: Overview of test results

\begin{tabular}{|c|c|c|c|c|c|}
\hline & $13 \mathrm{kA}$ & $4-7 \mathrm{kA}$ & $600 \mathrm{~A}$ & $120 \mathrm{~A}$ & $60 \mathrm{~A}$ \\
\hline 1 & $0.8 \pm 0.3$ & $0.4 \pm 0.2$ & $0.4 \pm 0.2$ & $0.6 \pm 0.2$ & $3 \pm 1.3$ \\
\hline 2 & $1 \pm 0.2$ & $3 \pm 1.5$ & $2.1 \pm 0.3$ & $2.5 \pm 0.7$ & $10.8 \pm 1.9$ \\
\hline 3 & $1.5 \pm 0.9$ & $2.3 \pm 1.3$ & $1.7 \pm 0.7$ & $4.5 \pm 2.0$ & $8.2 \pm 3.2$ \\
\hline 4 & $1.4 \pm 0.6$ & $5.7 \pm 2$ & $5.0 \pm 0.6$ & $6.7 \pm 1.0$ & $14.8 \pm 3.2$ \\
\hline 5 & $0.4 \pm 0.5$ & $0.5 \pm 0.3$ & $0.5 \pm 0.3$ & $0.8 \pm 0.4$ & $3.3 \pm 1.5$ \\
\hline
\end{tabular}

1:Stab@ $\mathrm{I}_{0}, \quad 2:$ Noise@ $\mathrm{I}_{0}, \quad 3: \quad$ Stab@ $\mathrm{I}_{\mathrm{n}}, \quad 4:$ Noise@ $\mathrm{I}_{\mathrm{n}}$, 5:Reproducibility

The results, except for 13kA, show a non-negligible dispersion, which can be explained by the varying temperature and noise environment during the tests (different times of the day and of the year). An improvement in stability at $\mathrm{I}_{\text {nom }}$ of at least $3 \mathrm{x}$ is expected after deployment of the temperature correction algorithm.

Modifications in the control loop and a better setup led to a 2-5x improvement in noise performance, either as a lower steady-state noise or in the elimination of level related noise regions.

The tests of the $13 \mathrm{kA}$ converters, the highest precision type, were in fact the most successful with the least problems [5]. Probably due to the emphasis and priority allocated during the early design and production phases.

\section{SHORT-CIRCUIT TESTS IN THE TUNNEL}

The short circuit tests were particularly important to validate the installation in the tunnel. However, the absence of an inductive load made precision tests impossible and only functional tests were carried out.

Nevertheless, the short-circuit tests were a good opportunity to characterize the environment in which the converters will work e.g. air and water temperature variations. The measurements carried out during the short-circuit tests in the different LHC galleries led to the identification of some air-conditioning and water cooling problems, which could then be corrected in good time.

\section{FINAL COMMISSIONING TESTS}

The original strategy aimed at a completely finished and calibrated converter at the end of the integration tests. This was not possible, except for $13 \mathrm{kA}$, due to the above mentioned drift and temperature problems and the converters were installed uncalibrated. The first high- precision (ppm level) calibration therefore takes place now in the tunnel and then with an increased importance. This campaign requires well established, stable conditions to be the starting point of the long-term monitoring. The final evaluation of noise and stability operating with its definitive load is carried out at the same time.

The calibration and testing in the tunnel has to be done with purpose-built equipment due to the safety restrictions imposed by the very high energy stored in the load. In particular, the calibration of the $120 \mathrm{~A}$ and $60 \mathrm{~A}$ converters requires the connection of a reference DCCT in the output leads of the converter.

Only a limited comparison can be made between the calibration data from the final commissioning and the data from the integration tests, but it confirms the uncertainty caused by the temperature problems. The real starting point is after the implementation of the correction algorithm, from which point the calibrations become quasi temperature independent

In conclusion, the deployment of the correction algorithm and the first tunnel calibration is the starting point for monitoring the converters' long-term highprecision performance. It also helps refining the calibration procedures and verifying the special calibration equipment used for tunnel calibrations.

\section{CONCLUSIONS}

- The multi-level test strategy adopted proved very successful in uncovering both design and production problems. The final commissioning has as a result been easier.

- The rejection rate of $>5 \%$ for DCCTs and 3-4\% for FGCs at reception tests at CERN demonstrates the limitations of factory tests.

- The short-circuit tests in the tunnel were useful functional tests and revealed the real environment in which the high performance must be achieved.

- The final commissioning tests have just started and the first results are promising.

\section{REFERENCES}

[1] LHC Design report v.1, p. 278, CERN-2004-003-V-1, June 2004

[2] G. Fernqvist, B. Halvarsson, J. Pett, J. Pickering, “A novel current calibration system up to 20kA", IEEE Trans. Instrum. Meas., vol. 52, pp. 445-448, Apr. 2003

[3] M. Bastos, High precision testing of LHC 4-7kA power converters, CERN Technical Note AB-POTN2007-03, EDMS 844606

[4] M. Bastos et al, Developments in high-precision aspects of power converter control for LHC, ibid.

[5] G. Hudson, High-precision integration tests of the 13 kA LHC power converters, CERN Technical Note AB-PO-TN2007-06, EDMS 847748 